\title{
Early impact of rotavirus vaccination in a large paediatric hospital in the UK
}

\author{
D. Hungerford ${ }^{a, b, *}$, J.M. Read ${ }^{c}$, R.P.D. Cooke ${ }^{d}$, R. Vivancos ${ }^{b}$, \\ M. Iturriza-Gómara ${ }^{a}$, D.J. Allen ${ }^{e}$, N. French $^{a}$, N. Cunliffe ${ }^{a, d}$ \\ a Institute of Infection and Global Health, University of Liverpool, Liverpool, UK \\ ${ }^{\mathrm{b}}$ Field Epidemiology Services, Public Health England, Liverpool, UK \\ ${ }^{c}$ CHICAS Group, Lancaster Medical School, Faculty of Health and Medicine, Lancaster University, Lancaster, UK \\ 'Department of Microbiology, Alder Hey Children's NHS Foundation Trust, Liverpool, UK \\ e Virus Reference Department, Public Health England, Colindale, London, UK
}

\section{A R T I C L E I N F O}

\section{Article history:}

Received 26 October 2015

Accepted 7 December 2015

Available online 31 December

2015

\section{Keywords:}

Epidemiology

Healthcare-associated infection

Rotavirus

Vaccination

CrossMark

\section{$S U M M A R Y$}

The impact of routine rotavirus vaccination on community-acquired (CA) and healthcareassociated (HA) rotavirus gastroenteritis (RVGE) at a large paediatric hospital, UK, was investigated over a 13-year period. A total of 1644 hospitalized children aged 0-15 years tested positive for rotavirus between July 2002 and June 2015. Interrupted time-series analysis demonstrated that, post vaccine introduction (July 2013 to June 2015), CA- and HA-RVGE hospitalizations were 83\% [95\% confidence interval $(\mathrm{Cl}): 72-90 \%)$ and $83 \%$ (95\% $\mathrm{Cl}: 66-92 \%]$ lower than expected, respectively. Rotavirus vaccination has rapidly reduced the hospital rotavirus disease burden among both CA- and HA-RVGE cases.

(C) 2016 The Healthcare Infection Society. Published by Elsevier Ltd. All rights reserved.

\section{Introduction}

Prior to the introduction of routine vaccination, rotavirus was the most frequent cause of severe gastroenteritis in children aged $<5$ years worldwide. ${ }^{1}$ In the UK, rotavirus gastroenteritis (RVGE) was estimated to be responsible for $45 \%$ and $20 \%$ of acute gastroenteritis hospitalizations and emergency department attendances in children aged $<5$ years, respectively. ${ }^{2}$ Rotavirus is also an important cause of healthcare-

\footnotetext{
* Corresponding author. Address: Institute of Infection and Global Health, Ronald Ross Building, 8 West Derby Street, Liverpool L69 7BE, UK. Tel.: +44 (0)151 7959609.

E-mail address: d.hungerford@liverpool.ac.uk (D. Hungerford).
}

associated (HA) gastroenteritis; among children at a large paediatric hospital, UK, rotavirus was detected by reverse transcription-polymerase chain reaction (RT-PCR) in $43 \%$ of community-acquired (CA) and in $31 \%$ of $\mathrm{HA}$ gastroenteritis cases. $^{3}$

Several European countries have introduced rotavirus vaccine into their childhood immunization programmes, with effectiveness against RVGE hospitalizations estimated at $>80 \% .^{4}$ In July 2013 the UK introduced the live-attenuated, two-dose oral monovalent rotavirus vaccine (Rotarix ${ }^{\mathrm{TM}}$, GlaxoSmithKline Biologicals S.A., Rixensart, Belgium) with doses given at two and three months of age. ${ }^{5}$ Vaccine uptake for a completed course reached $89 \%$ by June $2015 .^{6}$ Early impact studies in the UK suggested a large reduction $(77 \%)$ in 
Table I

Rotavirus gastroenteritis (RVGE) hospitalizations at Alder Hey among children 0-15 years of age, pre and post rotavirus vaccine introduction

\begin{tabular}{|c|c|c|c|c|c|c|}
\hline \multirow[t]{2}{*}{ Variable } & \multirow{2}{*}{$\begin{array}{l}\text { Yearly mean no. of } \\
\text { hospitalizations (range) } \\
\text { pre vaccine introduction, } \\
\text { July } 2002 \text { to June } 2013^{\text {a }}\end{array}$} & \multicolumn{2}{|c|}{$\begin{array}{l}\text { No. of hospitalizations post } \\
\text { vaccine introduction, } \\
\text { July } 2013 \text { to June } 2015\end{array}$} & \multirow[t]{2}{*}{ Risk ratio $(95 \% \mathrm{Cl})$} & \multirow[t]{2}{*}{$\begin{array}{c}\text { Percentage decline in } \\
\text { hospitalizations }(95 \% \mathrm{Cl})^{\mathrm{b}}\end{array}$} & \multirow[t]{2}{*}{$P$-value } \\
\hline & & Year 1 & Year 2 & & & \\
\hline $\begin{array}{l}\text { Overall } \\
\text { Age (years) }\end{array}$ & 145 (109-191) & 22 & 30 & $0.18(0.11-0.30)$ & $82(70-89)$ & $<0.001$ \\
\hline$<2$ & $111(88-145)$ & 17 & 16 & $0.16(0.10-0.26)$ & $84(74-90)$ & $<0.001$ \\
\hline $2-4$ & $22(15-32)$ & 2 & 13 & $0.31(0.14-0.62)$ & $69(38-86)$ & 0.017 \\
\hline $5-15$ & $12(6-20)$ & 2 & 1 & - & - & - \\
\hline CA-RVGE & $108(83-150)$ & 18 & 19 & $0.17(0.10-0.28)$ & $83(72-90)$ & $<0.001$ \\
\hline HA-RVGE & $37(18-58)$ & 4 & 11 & $0.17(0.08-0.34)$ & $83(66-92)$ & $<0.001$ \\
\hline
\end{tabular}

laboratory-confirmed rotavirus infections in vaccine ageeligible infants. ${ }^{7}$ However, no impact on $\mathrm{HA}$ infection has yet been described. Understanding the impact of rotavirus vaccination on both CA- and HA-RVGE cases may have implications for both hospital infection control and bed management policies, and will help inform the evidence base for continued immunization in the UK.

This retrospective investigation aimed to quantify the impact of rotavirus vaccination on HA- and CA-RVGE cases at the same children's hospital as our prospectively conducted study from the pre-vaccine period. ${ }^{3}$

\section{Methods}

\section{Study setting}

The study was conducted at Alder Hey Children's NHS Foundation Trust, Liverpool, UK (Alder Hey). Alder Hey provides primary, secondary, and tertiary care facilities for $>200,000$ children each year and has $\sim 240$ inpatient beds. General medicine, general surgery, and a range of specialist services including critical care, oncology, cardiology, and neurosurgery are provided; there is also a large emergency department.

\section{Case definition}

Children aged between 0 and 15 years who were admitted with RVGE between July 2002 and June 2015, or those in whom RVGE developed after hospitalization, were eligible for inclusion. Testing for rotavirus was conducted on clinician request throughout the study period with no age restriction. RVGE was defined as rotavirus antigen detected by immunochromatographic test or by enzyme immunoassay in a faecal specimen of a child with acute gastroenteritis. RVGE was considered HA if gastroenteritis developed $\geq 48 \mathrm{~h}$ after admission and there was no record of diarrhoea or vomiting on admission. Clinical and anonymized demographic data were collected for each participant, and included information on specimen date, admission date, age, and symptoms on admission. The pre- vaccine period was defined as July 2002 to June 2013 and the vaccine period was defined as July 2013 to June 2015.

\section{Statistical analysis}

To assess the impact of rotavirus vaccination on hospitalizations for CA- and HA-RVGE, an interrupted time-series methodology was used. First, monthly expected incidence of rotavirus hospitalizations was estimated by fitting a negative binomial regression model to pre-vaccine monthly incidence data, offset for total monthly admissions and adjusting for seasonality and secular trends using calendar month and rotavirus year (July to June), respectively. ${ }^{8}$ This model was used to predict the counterfactual numbers of RVGE hospitalizations (in the absence of vaccination) for the vaccine period, where the impact of vaccination is expressed by the difference between the counterfactual expectation and observed number of hospitalizations. To quantify change in the number of RVGE hospitalizations by the introduction of the vaccine, a second model included a derived binary indicator variable for the post-vaccine period, enabling the computation of risk ratios (RR) and associated 95\% confidence intervals $(\mathrm{Cl})$. This second model offset for total monthly admissions and adjusted for month and rotavirus year. Percentage change in incidence was calculated as $100 \times(1-\mathrm{RR})$. The analysis was undertaken separately for CA- and HA-RVGE hospitalizations. To investigate the impact of routine vaccination on different age groups the analysis stratified overall RVGE hospitalizations by age group ( $<2$ years and $2-4$ years).

Demographic and clinical characteristics were compared between RVGE cases from the pre- and post-vaccine periods and between CA- and HA-RVGE cases. Continuous variables were tested by Student's $t$-test or Wilcoxon rank-sum test if not normally distributed and $\chi^{2}$-test or Fisher's exact test for categorical variables. All data handling and statistical analyses were performed using R Version 3.1.2 (R Development Core Team, Vienna, Austria).

\section{Ethical approval}

Ethical approval was provided by NHS Research Ethics Committee, South Central-Berkshire (Reference: 14/SC/1140). 


\section{Results}

A total of 1644 hospitalized cases of RVGE were documented between July 2002 and June 2015. CA cases accounted for $74.2 \%(N=1220)$ of all RVGE cases, $25.4 \%(N=418)$ cases were HA-RVGE, and $0.4 \%(N=6)$ did not meet the case definition for either HA or CA. In the pre-vaccine period there was a mean of 145 RVGE hospitalizations per year (range: 109-191), comprising 108 (83-150) CA and 37 (18-58) HA cases (Table I). In the first post-vaccine year (July 2013 to June 2014) there were 22 RVGE cases and in the subsequent year (July 2014 to June 2015) there were 30 RVGE cases. In the pre-vaccine period 25\% (range: 15-35\%) of RVGE cases were classified as HA compared with $29 \%(18 \%$ in $2013 / 14 ; 37 \%$ in $2014 / 15)$ in the vaccine period $(P=0.6)$.

There was an estimated $82 \%$ reduction in RVGE hospitalizations (95\% Cl: $70-89 \%)$ in the vaccine period, compared with what would have been expected in the absence of vaccination (Table I; Figure 1). Most of the decline occurred in vaccineeligible children aged $<2$ years $(84 \% ; 95 \% \mathrm{Cl}$ : $74-90 \%)$. A reduction of $69 \%(95 \% \mathrm{Cl}: 38-86 \%)$ was observed in children aged 2-4 years who were too old to have been vaccinated. There was an insufficient number of RVGE hospitalizations in children aged $>5$ years in the pre-vaccine era (mean per year: 12; range: $6-20$ ) to enable fitting of the regression model (Table I). The magnitude of reduction in hospitalizations in the vaccine period was similar in both CA-RVGE $183 \%$; $95 \% \mathrm{Cl}$ : 72-90\%) and HA-RVGE (83\%; 95\% Cl: 66-92\%) cases (Table ।; Figure 1).

The median age of pre-vaccine CA-RVGE cases [12 months; interquartile range (IQR): 7-23] was lower than the age of CARVGE cases from the vaccine period (23 months, IQR): 14-26; $P<0.001)$. The median age of HA-RVGE cases that occurred in the pre-vaccine period (nine months; IQR: 4-22) was nonsignificantly higher than that of HA-RVGE cases in the vaccine period (five months; IQR: $4-14 ; P=0.131$ ). The median age of CA-RVGE cases in the pre-vaccine period was significantly higher than that of HA-RVGE cases $(P<0.001)$, with this age difference even greater in the vaccine period $(P<0.001)$.

\section{Discussion}

Since the introduction in 2013 of routine rotavirus vaccination in the UK there has been a significant decline in hospitalizations for RVGE in this large paediatric hospital. The magnitude of reduction was similar for both CA- and HA-RVGE cases. Age-stratified analysis provided further evidence that the reduction in hospitalizations is highly likely to be due to the impact of vaccination as the largest reduction was observed in vaccine-eligible infants aged $<2$ years. Furthermore, as shown in other settings, there was an increase in age of RVGE cases post vaccine introduction. ${ }^{7,9}$ The observed reduction in vaccine-ineligible older age groups (2-4 years) was similar to that observed through national laboratory surveillance and may indicate an indirect effect of vaccination. ${ }^{7}$

We established that HA-RVGE cases were significantly younger than CA-RVGE cases. Furthermore, an increase in age in the vaccine period was observed among CA-RVGE cases but this was not observed among HA-RVGE cases. Similar age profiles were also observed at a paediatric hospital in Greece following vaccine introduction. ${ }^{10}$ These data suggest that hospitalized infants aged $<1$ year remain at risk of developing HA-RVGE even among highly vaccinated populations, possibly through direct or indirect exposure to rotavirus from older children and adults.

Our study relied upon rotavirus antigen testing in stool, which is known to be a better predictor of symptomatic rotavirus disease than PCR-based methods; interpretation of a positive PCR result is rendered difficult because of the high frequency of asymptomatic rotavirus shedding in young

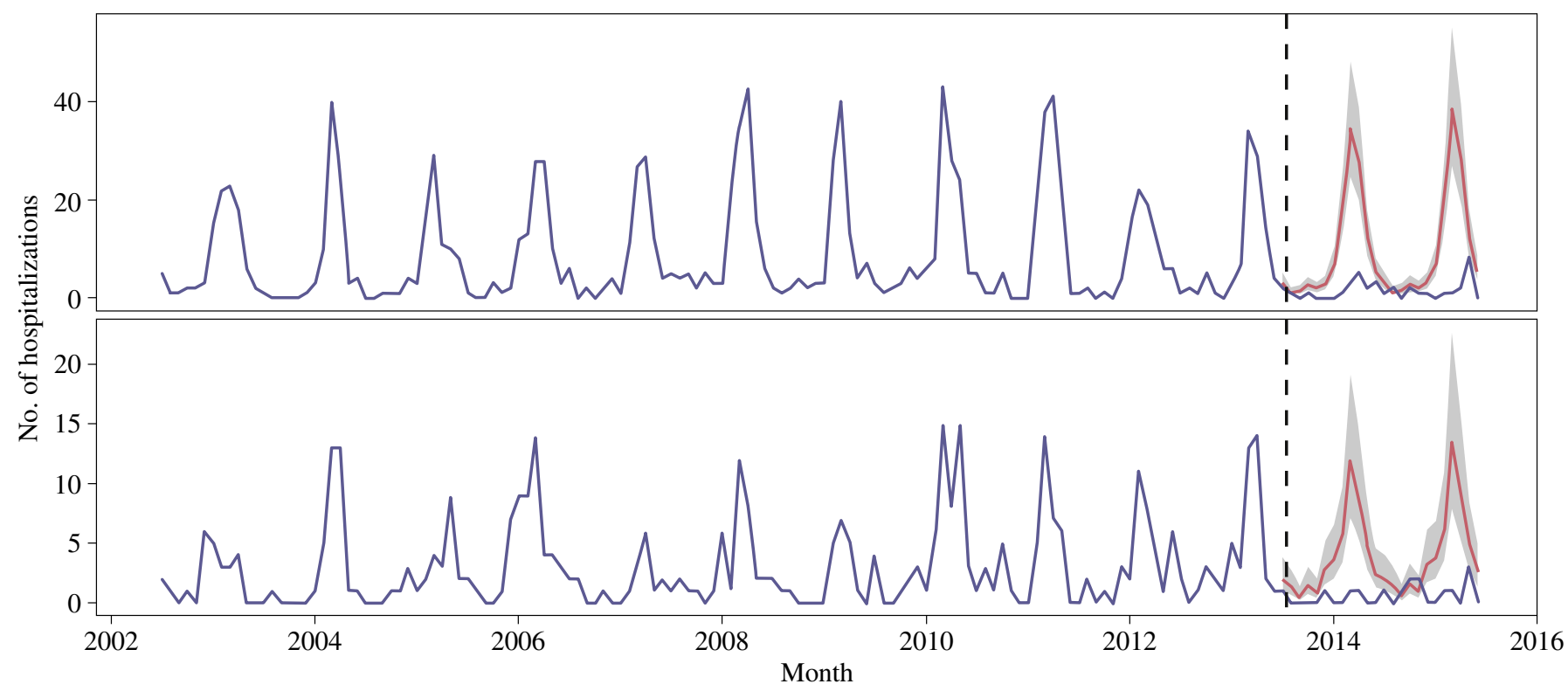

Figure 1. Community-acquired (upper graph) and healthcare-associated (lower graph) rotavirus gastroenteritis (RVGE) hospitalizations at Alder Hey, July 2002 to June 2015. The blue line is the observed incidence of RVGE; the red line is the expected incidence; the grey shading represents the $95 \%$ confidence intervals for the expected incidence. The black hashed line represents the introduction of rotavirus vaccine in the UK in July 2013. 
children, necessitating the development of a real-time PCR cut-off to define symptomatic infection. ${ }^{11}$ Therefore, it is likely that the cases in this study represent clinical disease. Although rotavirus vaccination cannot be definitively established as the cause of the observed reduction in RVGE hospitalizations due to the ecological nature of this study, there are additional factors suggesting vaccine impact. The reduction in CA- and HA-RVGE cases in the vaccine period has not been mirrored by a similar decline in respiratory syncytial virus (RSV) infection, another viral pathogen that predominately affects young children in winter; indeed, the incidence of HA-RSV infection has remained stable whereas RSV infection has increased in the community (data not shown). There were also no major changes in hospital infection prevention and control policies during the period of study. Finally, our study examined two post-vaccine seasons and took into account long-term seasonal and annual trends.

This study has demonstrated that, since the introduction of routine rotavirus vaccination in the UK, in addition to a marked decline of CA-RVGE cases there has been a similar fall in HARVGE hospitalizations. The reduction in RVGE cases is expected to save bed-days and reduce the burden on infection control teams with potential for both clinical and economic benefit.

\section{Acknowledgements}

The authors acknowledge the contribution of F. Hardiman, all staff in the microbiology department and K. Edwardson from Alder Hey Children's NHS Foundation Trust.

\section{Conflict of interest statement}

N.C., N.F., M.I.G., R.V. and D.H. are in receipt of research grant support from GlaxoSmithKline (GSK) Biologicals (EPI Rota-048); M.I.G. is in receipt of research grant support from Sanofi Pasteur-MSD (SPMSD); N.C. has received honoraria for participation in GSK Rotavirus Vaccine Advisory Board Meetings.

\section{Funding sources}

This study is supported by GlaxoSmithKline Biologicals SA (EPI Rota-048) and the University of Liverpool. GlaxoSmithKline Biologicals SA was provided the opportunity to review a preliminary version of this manuscript for factual accuracy but the authors are solely responsible for final content and interpretation. The authors received no financial support or other form of compensation related to the development of the manuscript. R.V. receives a salary contribution from NIHR Health Protection Research Unit in Emerging and Zoonotic Infections; R.V., M.I.G. and D.J.A. also receive salary contributions from the NIHR Health Protection Research Unit in Gastrointestinal Infections.

\section{References}

1. Tate JE, Burton AH, Boschi-Pinto C, Steele AD, Duque J, Parashar UD. 2008 estimate of worldwide rotavirus-associated mortality in children younger than 5 years before the introduction of universal rotavirus vaccination programmes: a systematic review and meta-analysis. Lancet Infect Dis 2012;12:136-141.

2. Harris JP, Jit M, Cooper D, Edmunds WJ. Evaluating rotavirus vaccination in England and Wales. Part I. Estimating the burden of disease. Vaccine 2007;25:3962-3970.

3. Cunliffe NA, Booth JA, Elliot C, et al. Healthcare-associated viral gastroenteritis among children in a large pediatric hospital, United Kingdom. Emerg Infect Dis 2010;16:55-62.

4. Soares-Weiser $\mathrm{K}$, Maclehose $\mathrm{H}$, Bergman $\mathrm{H}$, et al. Vaccines for preventing rotavirus diarrhoea: vaccines in use. Cochrane Database Syst Rev 2012;(11):CD008521.

5. Iturriza-Gómara M, Cunliffe N. Rotavirus vaccine: a welcome addition to the immunisation schedule in the UK. BMJ 2013;346:f2347.

6. Public Health England. National rotavirus immunisation programme: preliminary data for England, February 2014 to July 2015. Health Protection Report 2015;30:1-6. Available at: https:// www.gov.uk/government/uploads/system/uploads/attachment data/file/457925/hpr3015_rtvrs.pdf [last accessed October 2015].

7. Atchison CJ, Stowe J, Andrews N, et al. Rapid declines in age group-specific rotavirus infection and acute gastroenteritis among vaccinated and unvaccinated individuals within 1 year of rotavirus vaccine introduction in England and Wales. J Infect Dis 2015:jiv398.

8. Hungerford D, Vivancos R, French N, Iturriza-Gomara M, Cunliffe N. Ecological assessment of the direct and indirect effects of routine rotavirus vaccination in Merseyside, UK using data from multiple health systems: a study protocol. BMJ Open 2014;4:e006161.

9. Bar-Zeev N, Kapanda L, Tate JE, et al. Effectiveness of a monovalent rotavirus vaccine in infants in Malawi after programmatic roll-out: an observational and case-control study. Lancet Infect Dis 2015; 15:422-428.

10. Konstantopoulos A, Tragiannidis A, Fouzas S, et al. Burden of rotavirus gastroenteritis in children $<5$ years of age in Greece: hospital-based prospective surveillance (2008-2010). BMJ Open 2013;3(12).

11. Phillips G, Lopman B, Tam CC, Iturriza-Gomara M, Brown D, Gray J. Diagnosing rotavirus A associated IID: using ELISA to identify a cut-off for real time RT-PCR. J Clin Virol 2009;44:242-245. 